\title{
Low-threshold spiking interneurons perform feedback inhibition in the lateral amygdala
}

\author{
Çağrı Temuçin Ünal ${ }^{1}$ (D) Bengi Ünal ${ }^{1} \cdot$ M. McLean Bolton ${ }^{2}$
}

Received: 8 July 2019 / Accepted: 29 January 2020 / Published online: 6 March 2020

(C) The Author(s) 2020

\begin{abstract}
Amygdala plays crucial roles in emotional learning. The lateral amygdala (LA) is the input station of the amygdala, where learning related plasticity occurs. The LA is cortical like in nature in terms of its cellular make up, composed of a majority of principal cells and a minority of interneurons with distinct subtypes defined by morphology, intrinsic electrophysiological properties and neurochemical expression profile. The specific functions served by LA interneuron subtypes remain elusive. This study aimed to elucidate the interneuron subtype mediating feedback inhibition. Electrophysiological evidence involving antidromic activation of recurrent LA circuitry via basolateral amygdala stimulation and paired recordings implicate lowthreshold spiking interneurons in feedback inhibition. Recordings in somatostatin-cre animals crossed with tdtomato mice have revealed remarkable similarities between a subset of SOM+interneurons and LTS interneurons. This study concludes that LTS interneurons, most of which are putatively SOM+, mediate feedback inhibition in the LA. Parallels with cortical areas and potential implications for information processing and plasticity are discussed.
\end{abstract}

Keywords Basolateral amygdala $\cdot$ Somatostatin $\cdot$ Parvalbumin $\cdot$ Interneuron $\cdot$ Low-threshold spiking $\cdot$ Fast-spiking $\cdot$ Feedback inhibition

\section{Introduction}

Amygdala is a collection of different nuclei (Swanson and Petrovich 1998) that play cardinal functions in the acquisition and expression of fear responses (LeDoux 2000; Pape and Pare 2010; Gründermann and Lüthi 2015). The basolateral complex of the amygdala (BLA) and central amygdala (CeA) constitute the two main nuclei. The BLA is a collection of smaller cortex-like nuclei and is composed $75 \%$ of glutamatergic principal neurons that relay information to the output station of the amygdala, the central amygdala (CeA)

M. McLean Bolton

mclean.bolton@mpfi.org

Çağrı Temuçin Ünal

cagri.unal@tedu.edu.tr

Bengi Ünal

bengi.unal@tedu.edu.tr

1 Department of Psychology, Comparative Cognition Laboratory, TED University, Ziya Gokalp Caddesi No. 48 06420, Kolej Cankaya, Ankara, Turkey

2 Disorders of Neural Circuit Function, Max Planck Florida Institute for Neuroscience, Jupiter, FL 33458, USA
(McDonald 1992). These BLA glutamatergic principal neurons exhibit morphological and electrophysiological similarities with their cortical counterparts (Faber et al. 2001).

The remaining population $(\sim 25 \%)$ constitutes a neurochemically and electrophysiologically heterogeneous set of interneurons similar to that found in cortex (McDonald and Augustine 1993; Pare and Smith 1993; Spampanato et al. 2011; Bienvenu et al. 2012; McDonald and Augustine 2019) and are thought to play significant roles in determining the integration and plasticity of principal cell synaptic inputs and determining the dynamic range of their action potential firing output (Gaudreau and Pare 1996), thereby regulating information processing within the basolateral amygdala under normal and pathophysiological conditions (Letzkus et al. 2015; Fee et al. 2017; Krabbe et al. 2018).

The most salient parallels between BLA and other cortical regions with respect to their interneurons exist with respect to parvalbumin (PV) and somatostatin (SOM) positive interneurons. For instance, $\mathrm{PV}+$ interneurons form perisomatic baskets or axoaxonic synapses on principal neurons and constitute around $50 \%$ of the total interneuron population (McDonald and Mascagni 2001; McDonald and Bettette 2001; Rainnie et al. 2006; Vereczki et al. 2016; Butler et al. 2018) and play 
significant roles in fear learning (Lucas et al. 2016). Remarkably, these PV+interneurons exhibit a close correspondence in their electrophysiology to their cortical counterparts (Rainnie et al. 2006; Woodruff and Sah 2007). SOM positive interneurons constitute the other common interneuron population and they co-express markers such as neuropeptide $\mathrm{Y}$ (NPY) and calbindin, an expression profile similar to cortical SOM+interneurons (McDonald and Mascagni 2002; Truitt et al. 2009). Similar to cortex, SOM+interneurons selectively target the dendrites of BLA principal neurons (Muller et al. 2007).

However, there appears to be some interesting discrepancies. Calbindin positive/PV - interneurons which are putatively SOM+have been shown to receive intensive extra BLA input in anatomical studies (Unal et al. 2014) while anatomical and physiological data implicate $\mathrm{PV}+$ interneurons receiving a majority of their excitatory inputs from principal BLA neurons (Smith et al. 2000; Woodruff and Sah 2007; Unal et al. 2014; Spampanato et al. 2016). These findings implicate SOM+interneurons in feedforward inhibition while $\mathrm{PV}+$ interneurons appear to mediate feedback inhibition. The opposite scenario is thought to occur in other cortical regions (Urban-Ciecko and Barth 2016; Yavorska and Wehr 2016). It is still not clear whether these discrepancies involve the entire basolateral complex or whether there are subnucleus specific exceptions.

Importantly, BLA is composed of multiple smaller subnuclei that display hodological and functional distinctions (McDonald 1998; Pare et al. 2004). Among these nuclei, the lateral amygdala (LA) is the input station of the amygdala and it is thought to be the critical site of plasticity for the acquisition of fear memories (LeDoux 2000). In the current study, we aimed to identify the interneuron type that mediates feedback inhibition focusing specifically to LA to investigate whether our observations will parallel the observations made in the BLA overall. We have found a proportion of low threshold spiking (LTS) interneurons to exhibit reciprocal connectivity with principal cells of the LA while fast-spiking (FS) interneuron inputs were not reciprocated by principal neurons. Using transgenic cre lines crossed with tdtomato mice, we tested whether SOM and PV interneurons correspond to the electrophysiologically defined interneurons in our initial experiments. We have found that SOM+interneurons exhibiting the LTS profile were reciprocally connected with principal neurons. These findings suggest that a portion of LTS interneurons that are SOM+ mediate dback inhibition in the LA. Further experiments need to test to what extend this architecture extends to other BLA nuclei.

\section{Materials and methods}

Procedures were approved by the Institutional Animal Care and Use Committee of Max Planck Florida Institute, in compliance with the Guide for the Care and Use of Laboratory Animals (DHHS).

\section{Animals}

All experiments were done in adult male mice (2-4 months old). C57BL/6J mice were used for the initial experiments. For targeted somatostatin interneuron recordings, SOMCre (B6N.Cg-Ssttm2.1(cre)Zjh/J) and tdTomato (B6.CgGt(ROSA)26Sortm9(CAG-tdTomato)Hze/J) crosses were used. For targeted parvalbumin interneuron recordings, PV-Cre (B6N.129P2-Pvalbtm1(cre)Arbr/J) and tdTomato (B6.Cg-Gt(ROSA)26Sortm9(CAG-tdTomato)Hze/J) crosses were used. These mice were obtained from Jax Laboratories (Bar Harbor, ME). All mice were housed in AAALAC accredited animal facility with food and water ad libitum.

\section{Slice preparation}

Mice (PD 60-PD 120) were deeply anesthetized with isoflurane and transcardially perfused with $10 \mathrm{ml}$ of an ice cold solution containing (in $\mathrm{mM}$ ) 124 choline chloride, 2.5 $\mathrm{KCl}, 1.2 \mathrm{NaH}_{2} \mathrm{PO}_{4}, 3.3 \mathrm{MgCl}_{2} 26 \mathrm{NaHCO}_{3}, 10$ glucose, and $0.5 \mathrm{CaCl}_{2}$. The brains were then removed from the skull and blocks containing the amygdala were prepared. Subsequently, $300 \mu \mathrm{m}$ thick coronal sections containing the LA were obtained with a vibrating microtome using the same ice cold solution. The slices were then transferred to a holding chamber filled with an oxygenated (with $95 \% \mathrm{O}_{2}, 5 \% \mathrm{CO}_{2}$ ) artificial cerebrospinal fluid (ACSF) solution containing (in mM) $115 \mathrm{NaCl}, 3 \mathrm{KCl}, 1.25 \mathrm{NaH}_{2} \mathrm{PO}_{4}, 26 \mathrm{NaHCO}_{3}, 10$ glucose, $1 \mathrm{MgCl}_{2}, 2 \mathrm{CaCl}_{2}, 5$ sodium ascorbate, 3 sodium pyruvate and 2 thiourea at $32{ }^{\circ} \mathrm{C}$. Following $\sim 15 \mathrm{~min}$ of incubation at $32{ }^{\circ} \mathrm{C}$, the slices were transferred into another holding chamber containing the same solution at room temperature $\left(\sim 22^{\circ} \mathrm{C}\right)$.

\section{Electrophysiological recordings and analysis}

One hour or later (max $4 \mathrm{~h}$ ), one slice was transferred to a custom made recording chamber superfused with oxygenated ACSF (3-5 $\mathrm{ml} / \mathrm{min}$ ). LA neurons were visualized with an Olympus BX51WI (Center Valley, PA) microscope, equipped with infrared differential contrast optics. Under visual guidance, we obtained whole-cell recordings of LA neurons using pipettes (3-6 M $\Omega$ ) pulled from borosilicate glass capillaries and filled with a solution containing (in 
$\mathrm{mM}$ ): 145 potassium gluconate, $5 \mathrm{NaCl}, 10$ HEPES, 0.5 EGTA, 4 MgATP, $0.3 \mathrm{Na}_{2} \mathrm{GTP}$, for current clamp recordings and $120 \mathrm{Cs}$ methanesulfonate, $8 \mathrm{NaCl}, 15 \mathrm{CsCl}, 10$ TEACl, 10 HEPES, 0.5 EGTA, 10 QX-314, 4 MgATP and $0.3 \mathrm{Na}_{2} \mathrm{GTP}$ for voltage clamp recordings. The intracellular solutions were adjusted to a $\mathrm{pH}$ around $7.25 \pm 0.03$ and the osmolarity was adjusted to $290 \mathrm{mOsm} \pm 5$. In cases where the recording technique required a morphological analysis of the neurons, biocytin $(0.2 \%)$ was added into the intracellular solution. Current-clamp recordings were obtained with an Axoclamp 700B amplifier and digitized at $10 \mathrm{kHz}$ with a Digidata 1440A interface (Molecular Devices, Palo Alto, CA). Data acquisition ensued 5-10 min after whole cell access. During experiments, access resistances were monitored before the onset of each electrophysiological protocol. Cases where the access resistances exceeded $20 \mathrm{M} \Omega$ were discarded from analysis.

\section{LA circuitry analysis}

The investigation of intrinsic circuitry was multifaceted and involved various paired recording and electrical stimulation procedures. Preliminary analysis of intra-LA inhibition involved recording the responses of LA neurons to the stimulation of the basolateral amygdala (BL). In these experiments, recordings of LA neurons were done from a region corresponding to 1 to $1.4 \mathrm{~mm}$ posterior to Bregma. The stimulating electrodes were placed to a depth corresponding to $4-4.5 \mathrm{~mm}$ from the brain surface at the center of the BL (3-3.75 mm lateral to the midline). We typically used $100 \mu \mathrm{A}$ stimulation intensity unless otherwise indicated. Since information transfer between LA and BL is largely unidirectional, directly stimulating the BL would recruit descending LA fibers, antidromically stimulating the LA neurons (see Fig. 1a1 for a schematic representation). In these cases, the lidocaine derivative QX-314 $(10 \mu \mathrm{M})$ was added to the cesium based intracellular solution to block action currents in the recorded neurons that were kept at $0 \mathrm{mV}$ in voltage clamp to isolate the polysynaptic IPSCs that originate from local LA interneurons stimulated by other LA cells. In pilot experiments, a potassium-based intracellular solution was used instead of a QX-314 added cesium-based intracellular solution. In these cases, BL stimulation unequivocally led to action potentials LA neurons (not shown). Other pharmacological procedures were used to ascertain the recurrent nature of these evoked IPSCs (see results). These experiments were done in non-transgenic mice, somatostatin- and parvalbumin-tomato crosses, and GAD-GFPs to isolate the specific interneuron type that mediates recurrent inhibition.
A1
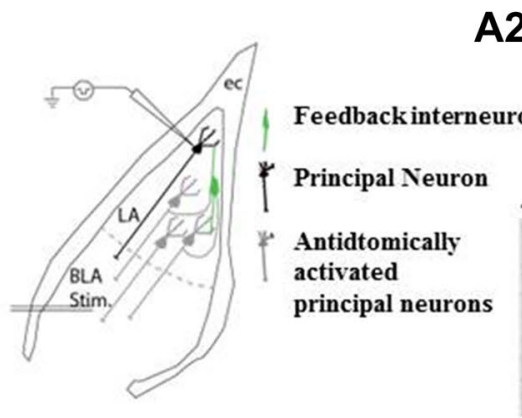

A2

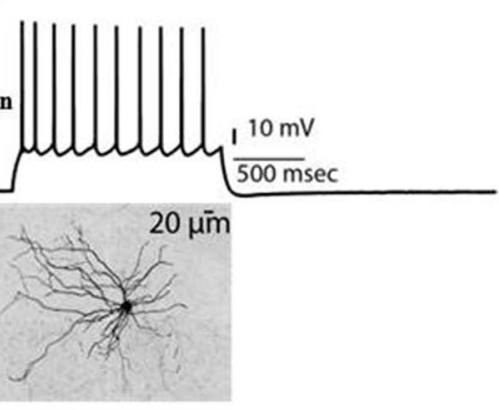

B1
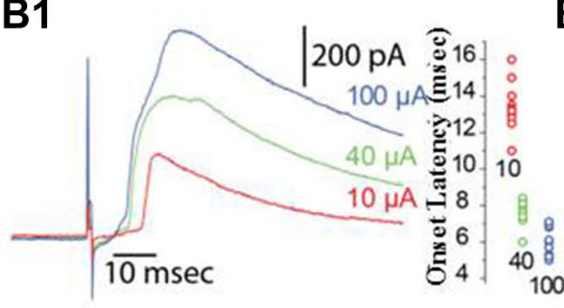

B2

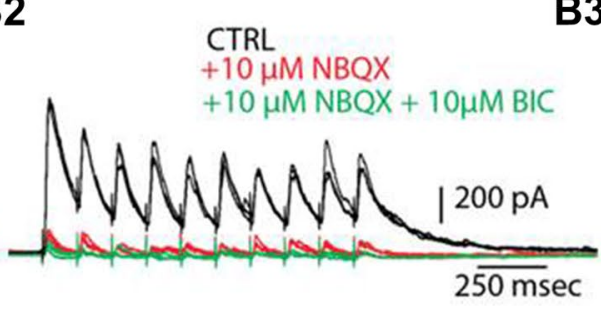

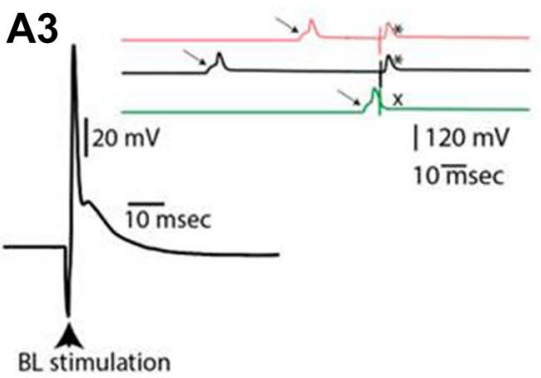

BL stimulation

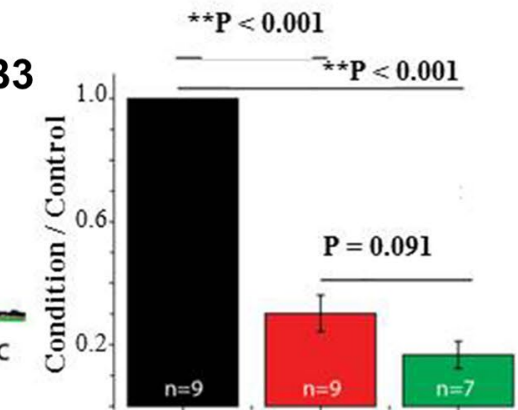

Fig. 1 Intranuclear inhibition in the LA. a Experimental protocol (a1), action potential discharge in an LA neuron in response to intracellular current injections and morphological features (a2), and an antidromic spike in the same neuron as a result of BL stimulation. Inset, three individual traces from a collision test (arrow, intracellular current evoked spike; asterisk, antidromic spike; $x$, collision) (a3). b Voltage clamp recordings $\left(\mathrm{V}_{\text {hold }}=0 \mathrm{mV}\right)$ reveal BL evoked polysynaptic IPSCs with a jitter in delays with increasing BL stimula- tion intensities (b1), and pharmacological profiling, revealing a high sensitivity to glutamatergic receptor signaling (b2 and b3). Values in color in b1 represent the BL stimulation intensity (b1). Representative data of a neuron recorded under different pharmacological conditions (b2). Quantification of pharmacological data (b3). Note that the majority of the inhibitory response disappears after the addition of AMPA-receptor blocker NBQX $(10 \mu \mathrm{M})$ 
In paired recordings, the potential presynaptic cell was stimulated at $50 \mathrm{~Hz}$ for $500 \mathrm{~ms}$ every $20 \mathrm{~s}$ and 15-20 traces in the potential postsynaptic neuron was averaged both for - 70 and - $55 \mathrm{mV}$ in current clamp for visualizing possible unitary EPSPs or unitary IPSPs (or polysynaptic IPSPs) respectively. In another set of paired recording experiments, the potential postsynaptic neurons were tested only - $70 \mathrm{mV}$ (for EPSPs only) in the presence of bicuculline $(10 \mu \mathrm{M})$. In cases where a monosynaptic connection was observed, a protocol involving $20 \mathrm{~Hz}$ stimulation of the presynaptic neuron for $500 \mathrm{~ms}$ (every $20 \mathrm{~s}$ ) was carried out and 40 to 50 such traces were acquired. All these tests were done with potassium based intracellular solutions in a bidirectional manner in current clamp. A typical strategy involved patching one neuron and sequentially recording other neurons with another pipette 2 for investigating the connectivities.

Rise time constants of synaptic events were calculated by fitting a single exponential between 10 and $90 \%$ of the maximal response. For paired recordings among monosynaptically connected pairs, the formal analysis was restricted to the last evoked synaptic event evoked by the post-tetanic stimulation. For polysynaptic pairs, IPSPs were detected using the event detection tool in clampfit. All available synaptic events for a particular pair (averages of the last evoked postsynaptic potentials in monosynaptically connected pairs and all detected IPSPs that could be detected using clampfit threshold detection) were averaged to obtain a single rise time constant value.

The distance between pairs of neurons tested ranged from 10 to 200 microns in all cases.

\section{Definition of feedback vs. non-feedback interneurons}

Among cells that satisfied the electrophysiological criteria for interneurons (i.e. a non-regular firing pattern and producing IPSPs in a postsynaptic neuron), those where at least 4 potential principal neuron presynaptic partners were probed were classified either as feedback or non-feedback interneurons depending on whether they received feedback connections from principal neurons (Fig. 4). This number is based on connectivity ratios between principal neurons and feedback interneurons in the BLA (Woodruff and Sah 2007) and other cortical areas (Yavorska and Wehr 2016).

\section{Intrinsic physiology of interneurons}

Neurons were kept at $-70 \mathrm{mV}( \pm 3 \mathrm{mV})$ in current clamp with bias current injections. Graded square pulses $(-100$ to $200 \mathrm{pA}$ in $20 \mathrm{pA}$ increments) were applied every $5 \mathrm{~s}$ for $1.5 \mathrm{~s}$.
Input resistances of neurons were estimated from the linear portion of the voltage-current $(V-I)$ curve. The time constants were measured by fitting an exponential to the voltage pulse starting with the offset of the current injection to return to baseline.

Action potential threshold was considered as the point where the rising slope of voltage exceeded $10 \mathrm{mV} / \mathrm{ms}$ at rheobase.

Other firing properties measured were the maximum firing frequency and adaptation ratio. The former was defined as the maximum frequency reached within the range of square pulses applied from $-70 \mathrm{mV}$. The latter was calculated at half maximal firing as "interspike interval (ISI) for the last two spikes/ISI for the first two spikes".

\section{Biocytin visualization and immunohistochemistry}

Following recordings with biocytin in the pipette $(0.2 \%)$, slices were transferred to $4 \%$ paraformaldehyde for at least $24 \mathrm{~h}$ for fixation. For biocytin visualization, slices were incubated in 1:200 Cy3-conjugated streptavidin or Texas Red conjugated streptavidin (Invitrogen, Carlsbad, CA; Jackson ImmunoResearch Laboratories, West Grove PA) for 20-24 h. Slices were then transferred to a slide and mounted with Vectashield wet mounting medium (Vector Labs Inc., Burlingame CA).

Slices were fixed in 4\% PFA in $0.01 \mathrm{M}$ PBS overnight. Following PBS washes and permeabilization with $1 \% \mathrm{Na}-$ Borohydride, $10 \%$ Methanol and $3 \% \mathrm{H}_{2} \mathrm{O}_{2}$ in PBS, slices were incubated in 5\% BSA, 5\% Triton X-100 and 1:400 Streptavidin-AlexaFluor 488/594 conjugate overnight. For PV staining, mice were perfused with $4 \%$ PFA and brains kept in $4 \%$ PFA overnight at $4{ }^{\circ} \mathrm{C}$. $50 \mu \mathrm{m}$ sections were collected. After the same cleaning and permeabilization steps as mentioned above, blocking was done in a cocktail of $10 \%$ NDS, $2 \%$ BSA, $5 \%$ Triton X-100 overnight at $4{ }^{\circ} \mathrm{C}$. Sections were then incubated in 1:1000 Goat-Anti-PV antibody in PBS for $48-72 \mathrm{~h}$ at $4{ }^{\circ} \mathrm{C}$. Sections were transferred into 1:400 Donkey-Anti-Goat AlexaFluor488 for $4-6 \mathrm{~h}$ and then rinsed in PBS prior to mounting. The same procedures were applied for somatostatin staining where the Goat-Anti-PV antibody was replaced with 1:1000 Goat-Anti-Somatostatin antibody.

\section{Confocal laser scanning microscopy and morphological reconstructions}

Digital images of biocytin-filled neurons and immunostained sections were acquired with a Zeiss LSM780 confocal microscope. The digital images were used for the 3-D reconstruction of neurons using the NeurolucidaTM Software (MBF Bioscience, VT, USA). For purposes of clarity, 
some biocytin filled neuron images are inverted versions of a gray scale image.

\section{Statistical analysis of the data}

All data were analyzed using Origin 7.0 (Northampton, MA, USA) and SPSS (Chicago, IL).

\section{Results}

\section{BL Stimulation and inhibition in LA neurons}

The following set of experiments was conducted to investigate whether the current slice preparation method preserves the inhibitory circuitry within the LA. When LA principal neurons were recorded with a potassium based internal solution, BL nucleus stimulation resulted in antidromic spikes in $5 / 22$ ( $20 \%, 22$ cells obtained from 4 animals) of LA neurons, consistent with previous experiments (Samson et al. 2003; Fig. 1a3). Compared to synaptically evoked spikes, these spikes were insensitive to NMDA and AMPA receptor antagonists, had a sudden onset following stimulation, exhibited a fixed latency, collided with intracellular current evoked spikes and arose from the baseline (Fig. 1a3). In these experiments, the identity of principal neurons were mainly verified through intracellular current injections to evoke spikes, which exhibit adaptation in principal neurons (Fig. 1a2; Washburn and Moises 1992; Faber et al. 2001) and morphological verifications (Fig. 1a2, bottom).

Next, we sought to determine if the recruitment of principal projection neurons result in polysynaptic inhibitory events, IPSCs in this case, in other principal neurons. For this purpose, cesium-based intracellular solutions supplemented with the lidocaine derivative QX-314 $(10 \mu \mathrm{M})$ were used for recordings. This approach assured that the postsynaptic voltage gated sodium channels are blocked and recorded neurons were not directly affected by the BL stimulation. Responses at $0 \mathrm{mV}$ were considered to be purely inhibitory based on the reversal potential of glutamatergic transmission under our experimental conditions (see methods). These responses did not have a fixed latency, displayed earlier onsets with stronger stimuli (Fig. 1b1) and were largely inhibited by glutamatergic receptor blockade (Fig. 1b2), suggesting that BL interneuron axons (Bienvenu et al. 2012) contribution to the IPSCs we observed are minimal. Furthermore, the magnitude of inhibition (paired $t$ test, $p<0.05$ ) with glutamatergic receptor blocker reached a peak, and GABA-A receptor inhibition did not result in an extra blockade (Fig. 1 b2 and b3; paired $t$ test, $p>0.05$; 11 neurons recorded from 4 animals). These data suggest that the IPSC seen under these conditions were polysynaptic in nature and result due to intranuclear recruitment of interneurons. Nonetheless, the polysynaptic recruitment might also result from the polysynaptic activation of BL interneurons (Bienvenu et al. 2012). Hence, the current slice approach is permissive, but not immune to problems, for studying the mechanisms of intranuclear inhibition.

\section{Inhibition revealed with LA principal neuron pairs}

The following sets of experiments involving paired recordings were conducted to unequivocally illustrate that the IPSCs observed in the previous experiment stem from local LA circuitry.

We obtained 58 principal neuron pairs from 10 animals with drug free ACSF using K-gluconate based intracellular solutions. Please refer to "LA circuitry analysis" in the methods section for a description of paired recording strategies (refer to Fig. 2a for a brief description). We found a unidirectional monosynaptic connection in one of these cases $(\sim 1.72 \%)$ (Fig. 2 b, c). In this case, the monosynaptic EPSP barrage was followed by a hyperpolarization, possibly a polysynaptic inhibition.

Because tonic inhibition present in the LA (Marowsky et al. 2012) has the potential to mask small EPSPs, we carried out additional experiments with bicuculline $(10 \mu \mathrm{M})$ in the bath. An additional 153 pairs were recorded 15 animals where 3 unidirectional monosynaptic connections were observed (1.96\%). Hence, 211 pairs were recorded in total and only 4 monosynaptic connections were observed, corresponding to a $\sim 1.89 \%$ connectivity rate (not shown).

In contrast to the scarcity of monosynaptic connections (4 out of 211, groups combined), the incidence of polysynaptic inhibition was relatively high (9 out of 59 pairs tested corresponding to $\sim 15.25 \%$; Fig. $3 b$ ). As mentioned before, in one case, we observed a monosynaptic EPSP barrage before the occurrence of polysynaptic IPSPs. This pair was removed from the analysis of amplitude and onset analysis of polysynaptic IPSPs. The average peak IPSP amplitude for all traces acquired was $0.93 \pm 0.03 \mathrm{mV}$ (data from 8 pairs, 160 traces). Evidence for the polysynaptic nature of these connections comes from the irregularity of IPSP onsets in the postsynaptic neuron $(161 \pm 15 \mathrm{~ms}$; Fig. $3 \mathrm{a} 2)$ and the sensitivity of the postsynaptic IPSPs to glutamatergic receptor blockers (Fig. 3a1, bottom traces). The rise constants (Fig. 5d) of polysynaptic IPSPs were relatively slow (from 6 to $11 \mathrm{~ms}$ ), which guided us in later experiments (see below) to putatively determine the interneuron mediating these effects.

In short, these experiments revealed that polysynaptic IPSPs in the LA can be shown with paired recordings and the kinetics of these IPSPs have the potential to be utilized in finding the interneuron that mediates feedback inhibition. 


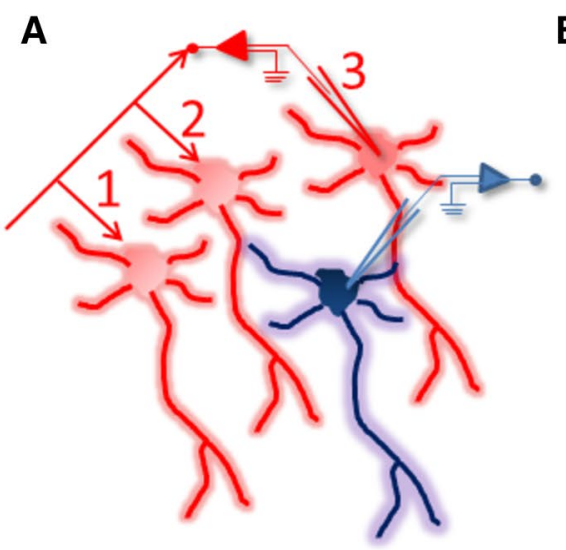

B

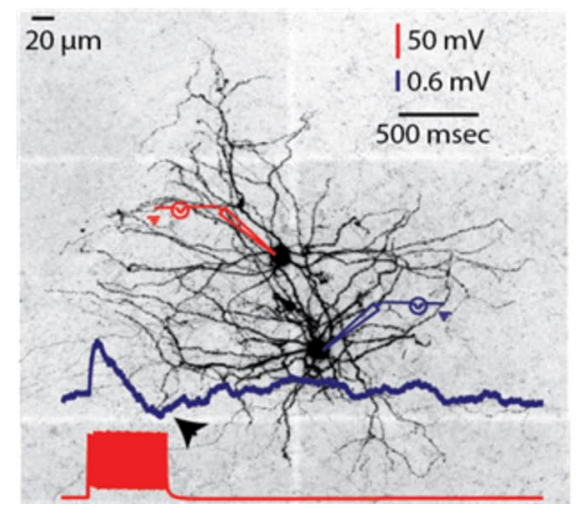

C

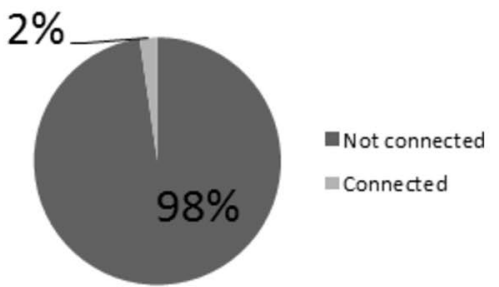

Fig. 2 Monosynaptic connectivity among LA principal neurons. a An illustration for serial connectivity search. In this example, an LA neuron is held throughout the recording (blue neuron) and synaptic partners are searched (red neurons) in a sequential manner. The numbers in the illustration indicate the order with which synaptic partners are searched. b A synaptically connected pair in a unidirectional manner.
$50 \mathrm{~Hz}$ stimulation of the presynaptic neuron (red neuron) triggers a barrage of EPSPs followed by a hyperpolarization (arrow), putatively a polysynaptic IPSP barrage. $\mathbf{c}$ Pie chart illustrating the ratio of connected pairs with and without bicuculline combined (4 out of 211 pairs; $1.89 \%$ combined)
A1

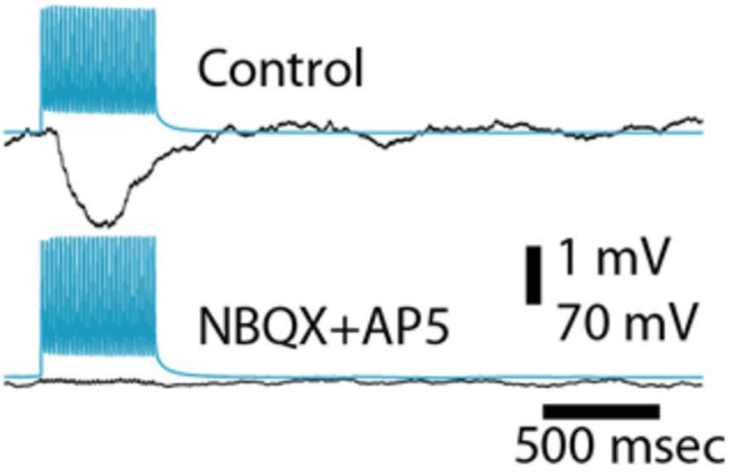

A2

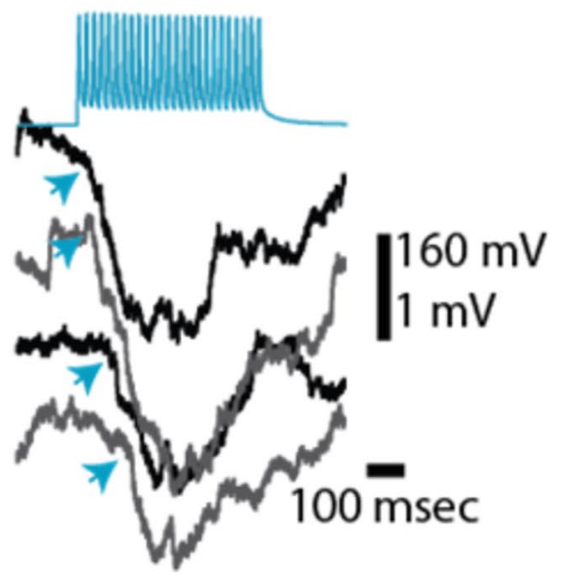

B

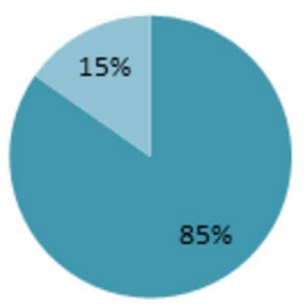

C1

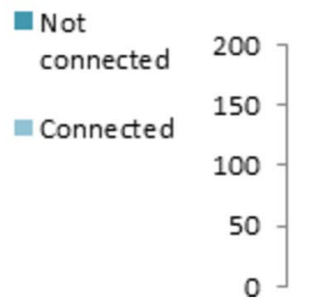

Onset Latency (msec)

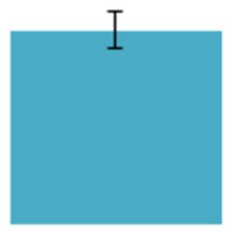

C2
Fig. 3 Polysynaptic inhibition observed in LA principal neuron pairs. a Recordings of polysynaptic IPSPs in LA principal neuron pairs. Postsynaptic inhibitory responses (black traces) to a $500 \mathrm{~ms}, 50 \mathrm{~Hz}$ stimulation of the other LA principal neuron (blue traces) (average of 50 traces; a1 top). The postsynaptic response dissipating as a result of bath application of CNQX $(10 \mu \mathrm{M})$ and AP-5 $(50 \mu \mathrm{M})(\mathbf{a} 1$, bottom).
Representative individual traces demonstrating the variability in the onset of polysynaptic IPSP barrages (a2). b Incidence of polysynaptic connections among principal LA neurons as illustrated by a bar chart. c Properties of polysynaptic IPSP barrages. Onset latencies (c1) and peak amplitudes (c2) 


\section{Interneuron-Principal neuron pairs: differences between feedback and non-feedback interneurons}

Experiments involving principal neuron-interneuron paired recordings were done to directly observe the interneuron type fulfilling the feedback inhibition function. Two things constitute the defining criteria of feedback interneurons: 1 -to receive excitatory inputs from the local collaterals of principal neurons; $2-$ to inhibit the principal neurons. To determine the complete profile of feedback interneurons within the LA microcircuitry, a comparison with non-feedback interneurons is necessary. To formulate a strong definition for non-feedback neurons, we considered any interneuron not connected to its 4 neighboring principal neurons as a "non-feedback" interneuron (Woodruff and Sah 2007). Other interneurons where a sufficient number of potential presynaptic partners were not probed were not taken into consideration. We mainly used a search approach until finding interneurons (Popescu and Pare 2010) and connections where at least 4 principal neurons were serially recorded and tested for bi-directional connectivity while an interneuron was being recorded (Fig. 4). In these experiments, we recorded 14 interneurons from 8 animals that satisfied the abovementioned criteria for differentiating "feedback" and "nonfeedback" interneurons. These neurons were easily classified as interneurons due to their electrophysiological dissimilarity to principal neurons (Fig. 5a, b, top traces) and the unitary IPSPs they generated in their postsynaptic principal neuron partners (20/35 for "feedback" interneurons; 25/32 for "non-feedback" interneurons; Fig. 5a, b, bottom traces). For each interneuron, we probed at least 4 potential excitatory presynaptic neurons. For seven of these interneurons, no principal excitatory cell synaptic partner was found and therefore these neurons are tentatively referred as nonfeedback interneurons (none of the 32 principal neurons tested for synaptic connectivity were found to trigger uEPSPs in these neurons). Interestingly, these "non-feedback" interneurons could be classified as conventional fast-spiking or stuttering fast-spiking interneurons based on their fast discharge rates and short-duration action potentials (Fig. 5). For the remaining 7 interneurons, we probed 35 potential excitatory presynaptic partners from principal cells. Overall, 13 out of the 35 principal neurons $(\sim 37 \%)$ were found to provide unitary EPSPs $(0.22 \pm 0.03 \mathrm{mV})$ to these "feedback" interneurons (Fig. 5b, right). Unlike their fast-spiking counterparts, these neurons had much lower action potentials thresholds (Fig. $5 c ; t(12)=7.252, p<0.0001$ ), wider action potentials (Fig. $5 \mathrm{~b} ; t(12)=4.083, p=0.0015$ ), they exhibited a voltage sag, indicative of an h-current (Fig. 5b), and they exhibited a continuum of spike frequency adaptation. Based on the fact that action potential threshold is the factor that clearly distinguishes feedback interneurons from non-feedback interneurons (Fig. 5c1), feedback interneurons are from now on referred as low-threshold spiking (LTS) feedback interneurons.

The average paired-pulse ratio (EPSP2/EPSP1 for two action potentials separated $50 \mathrm{~ms}$ apart) of these unitary EPSPs were $(1.28 \pm 0.14)$ (not shown). This facilitating pattern was also evident with stimulus trains of stimulations that ranged from 20 to $50 \mathrm{~Hz}$ in our connection search protocols (Fig. 4b, right). The rise and decay time constants for the uEPSPs were $4.43 \pm 0.76 \mathrm{~ms}$ and $15.43 \pm 6.47 \mathrm{~ms}$, respectively.

Conversely, IPSP rise kinetics between di-synaptic IPSPs and LTS feedback interneuron firing evoked IPSPs exhibited striking similarities (H $(4.15, p<0.0001$, see Fig. $5 \mathrm{~d}$ for

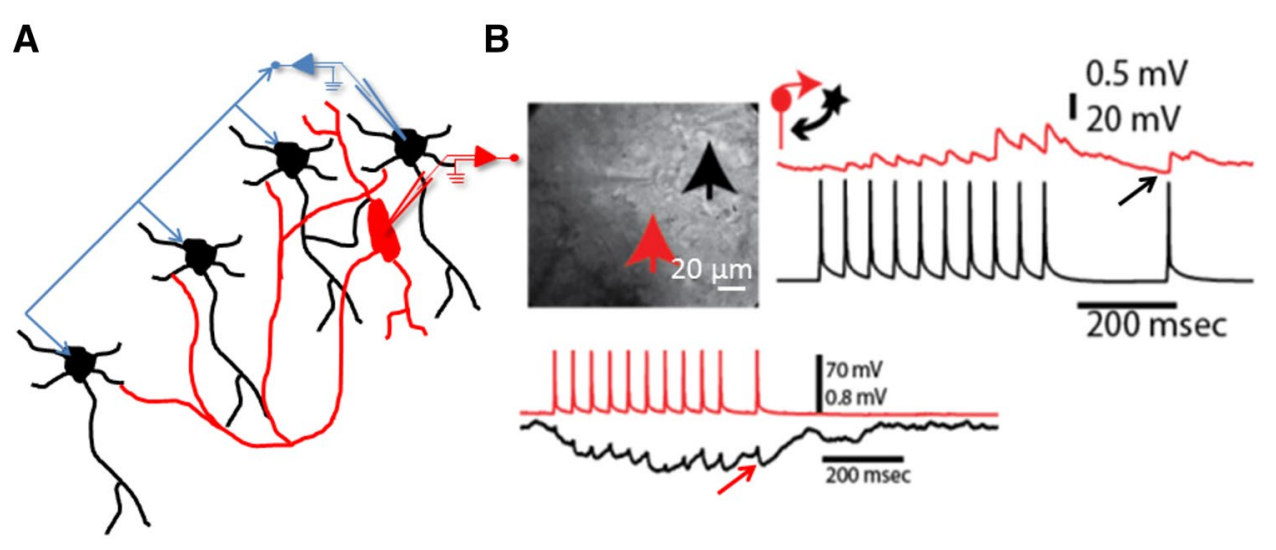

Fig. 4 Identification of feedback interneurons. a An illustration of a serial paired recordings which involves recording of an interneuron (shown in red) while probing at least four principal potential synaptic partners (shown in black) in a bidirectional manner. b A representative recording from a feedback interneuron bidirectionally connected to its principal neuron synaptic partner. The image in the inset illus- trates the distance between recorded neurons. For ease of illustration, the recorded neurons are shown with arrows colored correspondingly. Averaged electrophysiological traces from the recording showing connectivity in principal neuron $\longrightarrow$ feedback interneuron (top traces) and feedback interneuron $\longrightarrow$ principal neuron (bottom traces) direction 

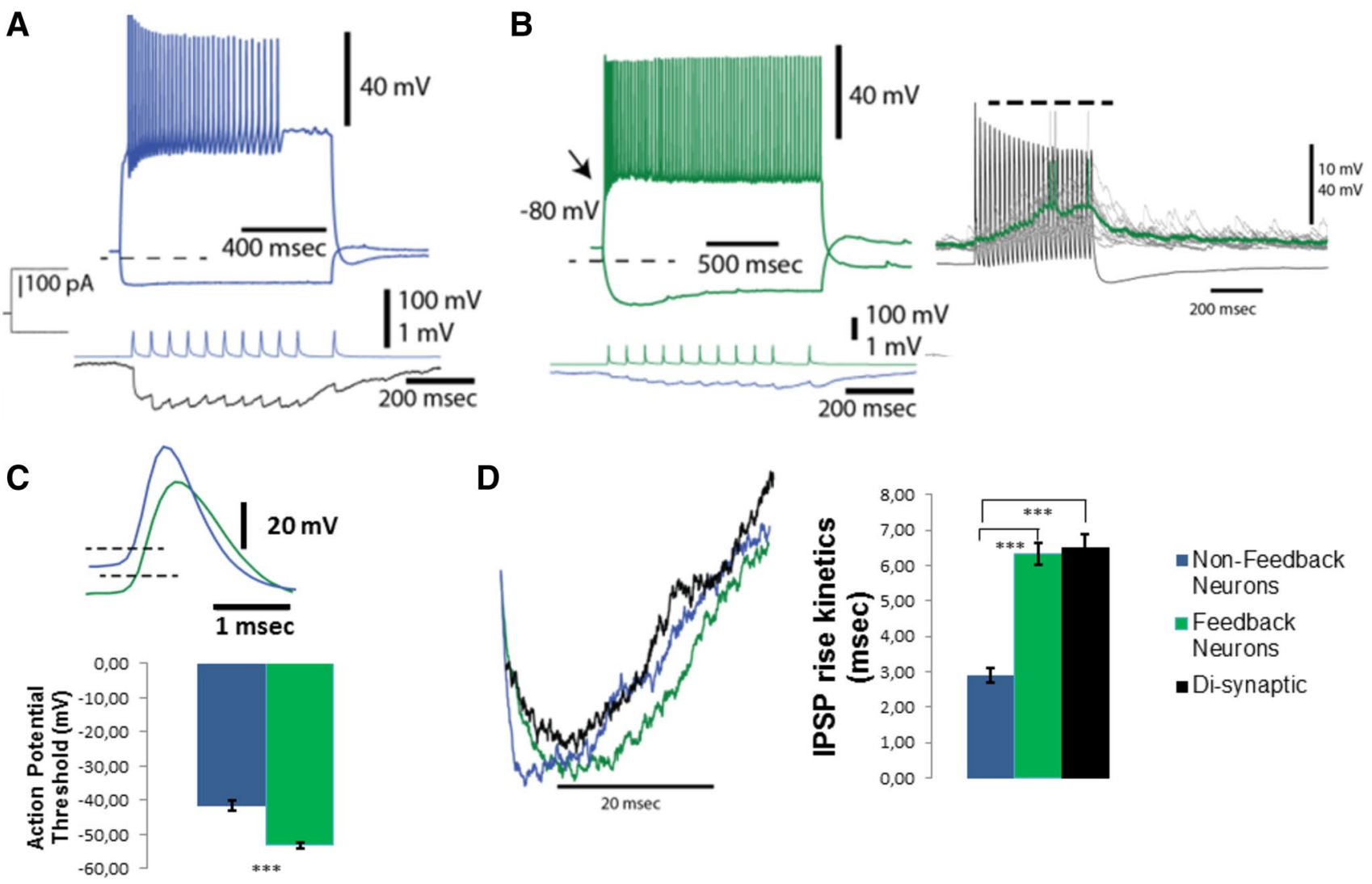

- Non-Feedback

Neurons

Feedback

Neurons

-Di-synaptic

Fig. 5 Feedback interneurons can be electrophysiologically defined as low-threshold spiking (LTS) interneurons. a Firing pattern of a nonfeedback interneuron (top panel) and IPSPs it elicits in a principal neuron (bottom panel, black trace, average of 20 raw traces). b Firing pattern of a feedback (LTS) interneuron (left, top panel) and IPSPs it elicits in a principal neuron (bottom panel, black trace, average of 20 raw traces). Black arrow on the top panel indicated lowthreshold spikes riding on a putative calcium plateau. On the right, responsiveness of the LTS interneuron to principal cell firing is illustrated. EPSP evoked spikes in the LTS interneuron are truncated. The dashed lines in A and B indicate the respective membrane potential values. c Comparison of representative action potentials in a feedback (green trace) and a non-feedback (blue trace) neuron. Note the difference in the width and threshold of the action potentials. Bar graph illustrating the difference in the action potential threshold of feedback and non-feedback interneurons (the same color coding as in the representative traces. $* * *$ indicates a significant difference with $p<0.000$ (C2). d Representative traces illustrating the similarity between the rise kinetics in LTS elicited IPSPs to those IPSPs seen during polysynaptic inhibition (color coding is the same for feedback and nonfeedback interneuron elicited IPSPs; black trace indicates an IPSP elicited as a result of polysynaptic input). Bar graph on the right summarizes statistical comparisons. $* * *$ indicates a significant difference with $p<0.000$ pairwise comparisons). This reinforced our thinking that the interneuron mediating the polysynaptic inhibition is the LTS interneuron (Fig. 5d). Specifically, the rise time constants of both disynaptic IPSPs and LTS evoked IPSPs ranged between $6-11 \mathrm{~ms}$ and largely overlapped $(6.50 \pm 0.39 \mathrm{~ms}$ for polysynaptic inhibition; $6.33 \pm 0.35 \mathrm{~ms}$ for LTS evoked unitary IPSPs) while nonfeedback interneuron evoked IPSP rise times had a range of $2-4 \mathrm{~ms}(2.89 \pm 0.20 \mathrm{~ms})$ and exhibited no overlap with former IPSPs.

\section{Further characterization of LTS feedback interneurons: Use of SOM- and PV-cre tomato animals}

To further characterize the interneuron type involved in feedback inhibition, we used SOM-Tomato and PV-Tomato animals in initial experiments to characterize their intrinsic physiology and synaptic connectivity (both paired 
recordings and BL-evoked EPSPs) and contrast these findings to the abovementioned results.

First, we intended to determine the overlap of PV and SOM within the LA, the main interest of the current project. To do that, we ran immunocytochemistry for PV in $3 \mathrm{SOM}$ tomato animals. While substantial overlap was observed within the BL with 53\% of SOM tomato neurons co-expressing PV (96 SOM tomato neurons counted from LA sections belonging to three mice), there was minimal overlap within the LA with only $3 \%$ of SOM tomato neurons exhibiting PV immunoreactivity (135 SOM tomato neurons counted from LA sections belonging to three mice) (Fig. 6). The overlap seen in the PV neurons in the BL might reflect transient developmental expression of SOM in PV neurons ( $\mathrm{Hu}$ et al. 2013) as SOM protein levels are observed to decrease as maturation proceeds (Forloni et al. 1990; Papadopoulos et al. 1993).

In our recordings, 6/9 PV interneurons ( 3 animals) could be regarded as classical fast spiking as evidenced by narrow spikes $(0.9-1.2 \mathrm{~ms})$ and high firing frequency $(60-100 \mathrm{~Hz})$ and they resembled the non-feedback interneurons from our recordings taken from wild type animals. The rest, exhibited spike frequency adaptation and some degree of depolarization block (Fig. 7a, top raw), in line with the heterogeneity observed in the BLA and other regions (Cauli et al. 2000; Rainnie et al. 2006; Woodruff and Sah 2007; Sosulina et al. 2010). Conversely, $4 \mathrm{SOM}+$ neurons out of $11 \mathrm{SOM}+$ neurons (four animals) exhibited a low action potential threshold comparable to the feedback LTS interneurons recorded in the previous experiment. In addition, these cells exhibited similarities with the LTS feedback interneurons in other respects such as the presence of spike frequency adaptation and a voltage sag indicative of an h-current. A minority (3/11) of SOM+ interneurons behaved like fast spiking interneurons (Fig. 7a, bottom raw).

When we electrically stimulated the BL using $100 \mu \mathrm{A}$ currents, we observed spikes in 5/11 SOM+interneurons (Fig. 8a, b, top). Interestingly, those interneurons were the ones with lowest action potential thresholds as revealed by an independent samples t-test between spiking versus nonspiking SOM+interneurons $[t(9)=9.47, p<0.0001]$. We tested 3 out of these $5 \mathrm{BL}$ stimulation responsive neurons utilizing paired recordings. In all of these cases, we found a presynaptic principal cell which also was innervated by the recorded SOM+interneuron (Fig. 8c, d). Conversely, 1/9 PV+ interneurons exhibited spiking to BL stimulation. When we used higher current intensities $(150 \mu \mathrm{A}$ and above), $\mathrm{PV}+$ interneurons exhibited small responses $(<5 \mathrm{mV})$. Unfortunately, we lost the BL-responsive PV+ interneuron before conducting paired recordings. Nonetheless, its responsiveness constitutes a strong evidence for the possibility that it receives inputs from principal LA neurons.

Table 1 summarizes the statistical comparisons of membrane properties (input resistance, action potential threshold, maximum firing frequency, adaptation ratio, and sag ratio) used in interneuron identification across different experiments. A one-way ANOVA has revealed a main effect on input resistance $[F(3,30)=10.211, p<0.000]$ and

\section{SOM Cre: tdTomato PVICC}
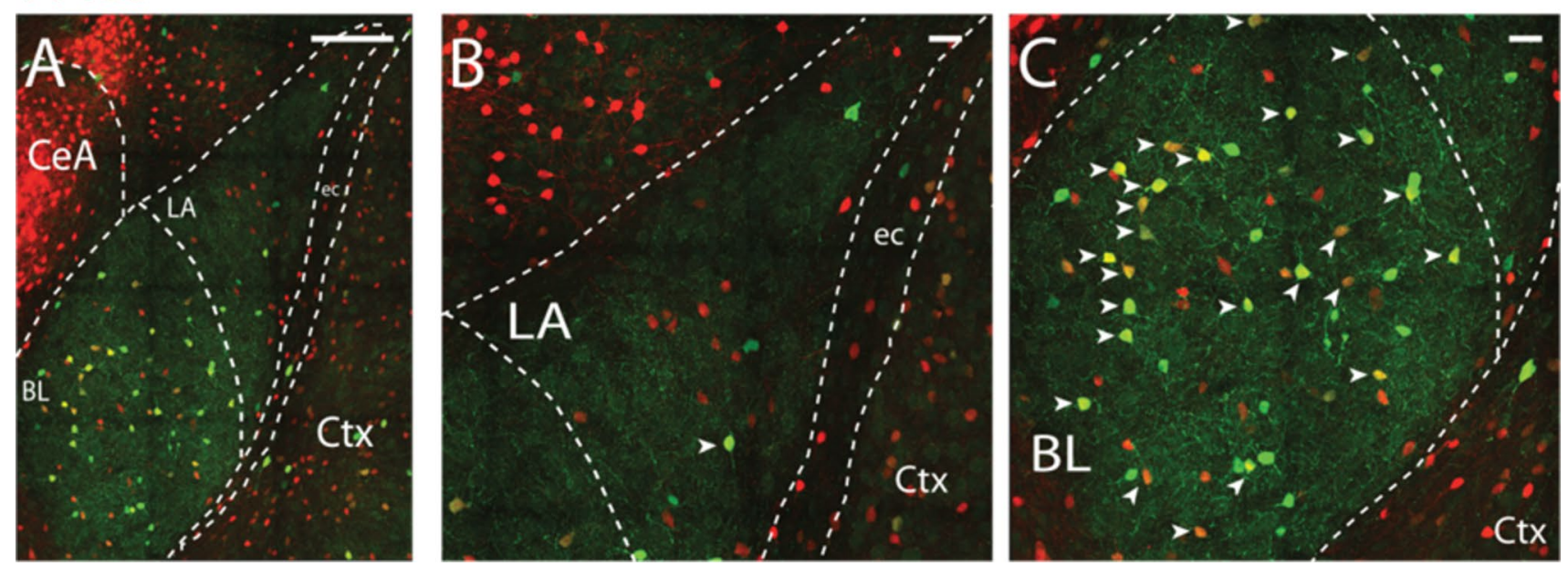

Fig. 6 Somatostatin (SOM)-tomato positive and Parvalbumin (PV) immune-positive interneurons constitute different populations of neurons within the LA but not within the BL. a Lower magnification image of the BLA complex illustrating PV immunoreactivity (green) in SOM-Cre:tdTomato mouse slice. b Magnified view of the LA from
$A$. Note the absence of co-labeling. C Magnified view of the BL from $A$. Note the abundance of PV and SOM co-expression in neurons. SOM-tomato/PV double positive neurons constituted $3 \%$ and $53 \%$ of LA and BL SOM-tomato neurons in the LA and BL respectively 
A
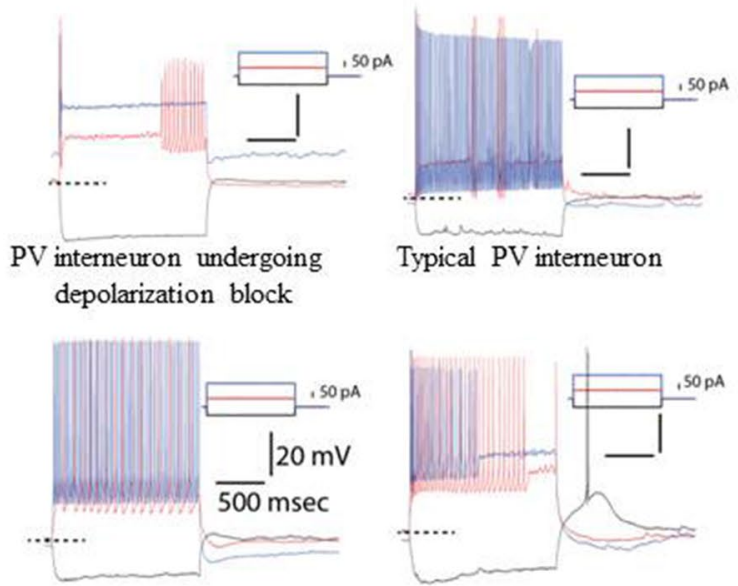

Fast-spiking SOM+ interneuron

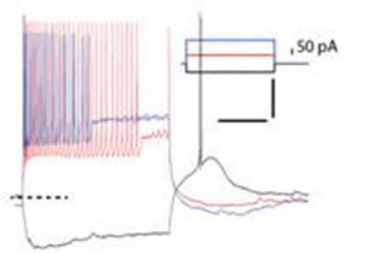

Typical SOM+

interneuron
B

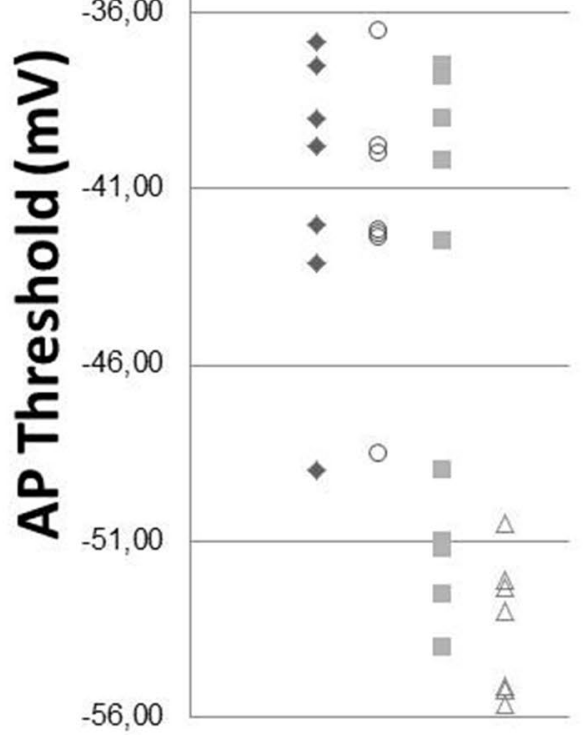

- PV interneurons

SOM interneurons

Feedback interneurons

Non-

Feedback interneurons
Fig. 7 a Two parvalbumin (PV) (upper panel) and two somatostatin (SOM) (lower panel) interneurons with different electrophysiological profiles. The examples on the right sides represent the more typical

adaptation ratios $[F(3,30)=6.082, p=0.002]$. LSD post hoc tests revealed that feedback interneurons had significantly higher input resistances from all other groups $(p<0.05$ for all comparisons) in addition to a significant difference between SOM+ and PV+ interneurons $(p=0.046)$. LSD post hoc tests revealed also that the feedback interneurons exhibited a higher spike frequency adaptation as compared to all other groups $(p<0.05)$. The inability of ANOVA to capture similarities between feedback and SOM+interneurons from different experiments likely signifies the fact that there is more than one type of SOM+interneuron as in other brain regions. In line with this notion, when feedback interneurons were compared to BL-responsive SOM+interneurons with independent samples t-tests, not a single difference emerged in any of the electrophysiological parameters reported while significant differences between BL-responsive $\mathrm{SOM}+$ interneurons and BL-unresponsive SOM+interneurons were observed along multiple dimensions including their input resistances $(p<0.01)$, adaptation ratios $(p<0.01)$, and action potential thresholds $(p<0.001)$ (see Table 1$)$. Last but not the least, when we compared specifically the BL-responsive SOM+interneurons to $\mathrm{PV}+$ interneurons, significant differences emerged with regards to input resistance $[t(12), 4.10, p<0.01]$, adaptation ratio $[t(12), 5.10$, $p<0.001]$, sag ratio $[t(12), 0.73, p<0.05]$, and action potential thresholds [ $t(12), 5.41, p<0.001]$ (see Table 1 ). electrophysiological profile for these populations. b Scatter plot of action potential thresholds for SOM and PV interneurons along with data from previous experiments

These results indicate that BL-responsive $\mathrm{SOM}+$ interneurons (which are also feedback SOM+interneurons) constitute a distinct population than BL-unresponsive $\mathrm{SOM}+$ and $\mathrm{PV}+$ interneurons.

\section{Discussion}

The current study aimed to elucidate the mechanisms of intranuclear feedback inhibition within the LA. The largely unidirectional information flow within the BLA complex and the preservation of feedback loops in the same rostro-caudal plane (Samson et al. 2003) makes the coronal slices feasible for studying feedback inhibition using BL-stimulation and paired recordings. Indeed, feedback inhibition was evident both using BL-evoked responses in LA principal neurons and paired recordings. Paired recordings between principal neurons have shown that this inhibition form is not uncommon $(15 \%)$ in this region.

We relied on certain parameters to identify the feedback interneuron in the LA circuitry. The first criterion entailed finding an interneuron that was reciprocally connected with the principal neurons as this is an identifying feature of feedback circuits. Secondly, we relied on the kinetic analysis of unitary IPSPs evoked by the interneurons in interneuron $\rightarrow$ principal neuron pairs and polysynaptic IPSPs observed during principal neuron $\longrightarrow$ principal neuron 
A

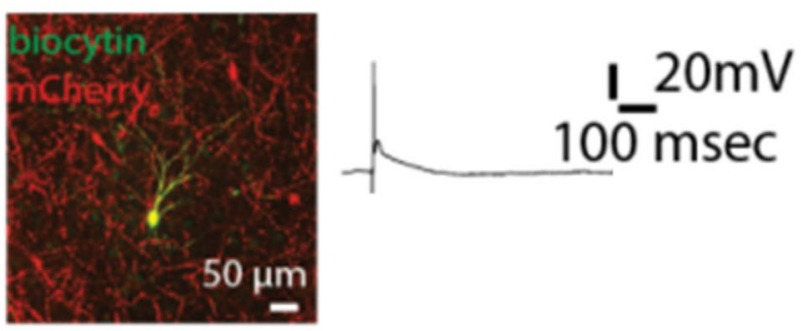

B

SOM+Responsiveness

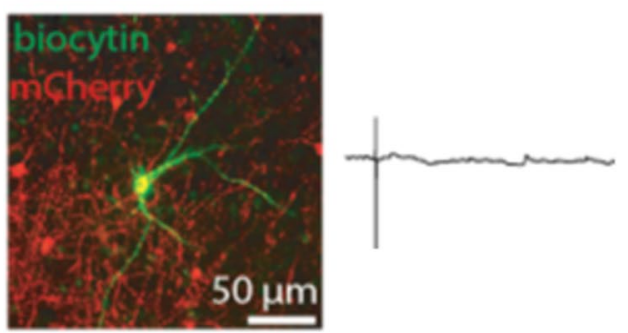

PV+ Responsiveness

C

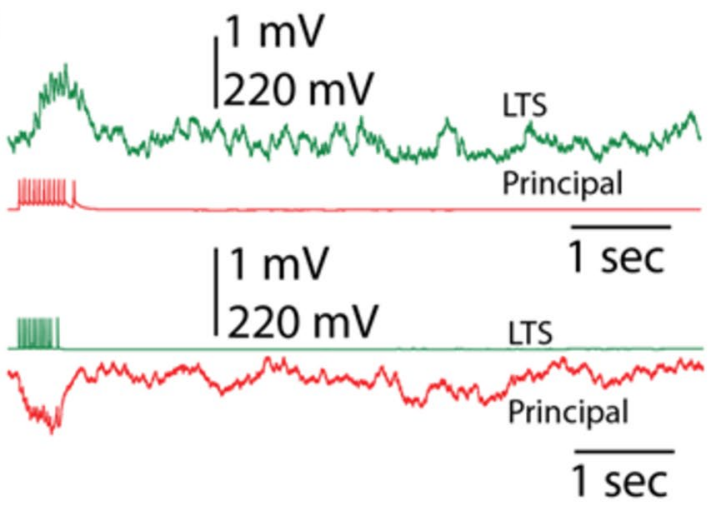

D

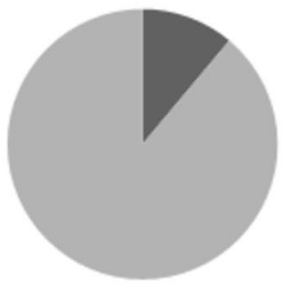

- Responsive

Non-responsive
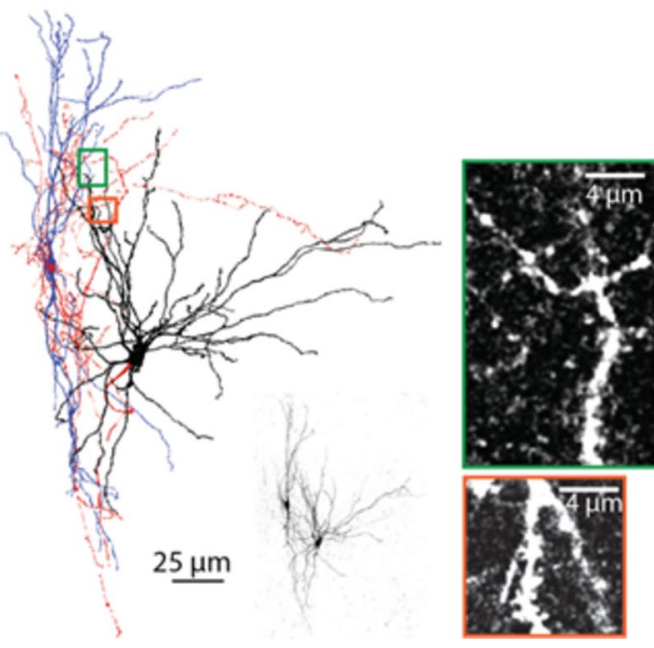

Fig. 8 Evidence for the feedback interneuron function of somatostatin $+(\mathrm{SOM}+)$ interneurons. a Representative responses of $\mathrm{SOM}+($ top) and parvalbumin+(PV+) (bottom) neurons to $\mathrm{BL}$ stimulation. Micrographs are on the left and electrophysiological traces are on the right. b Bar charts illustrating responsiveness rates as evidenced by spiking activity to BL stimulation (5/11 in

recordings. Based on these, this study concludes that polysynaptic IPSPs are elicited by interneurons that have lower action potential thresholds (LTS interneurons). When we analyzed these polysynaptic IPSPs, we relied on the event detection tool in clampfit program to dissect only those events that had a rising phase that could be explained with a single exponential and a decay phase after the peak. It is critical to note that this might have introduced a degree of bias in data analysis and could have masked the visualization of IPSPs coming from other sources. The LTS interneurons exhibited a continuum of spike frequency adaptation and voltage sag indicative of h-current, making them an electrophysiologically distinct group from fast spiking cells.
SOM + interneurons and 1/9 in PV interneurons). c Reciprocally connected SOM+interneuron and an LA principal cell. d Morphological reconstruction of the recorded pair in $\mathrm{C}$ (left), original biocytin images of the entire neurons (middle) and magnified images of putative contact sites (right) highlighted on the reconstruction

Experiments done in SOM-tdtomato and PV-tdtomato animals have revealed remarkable similarities between LTS and a proportion of SOM+ interneurons. These findings are in line with observations in other brain regions (Gibson et al. 1999; Beierlein et al. 2003; Goldberg et al. 2004). A limitation in our current approach entails the wide range in the distance among recorded pairs (10 to 200 microns). While it is possible that keeping a smaller distance could generate different results, this scenario is partially negated by our antidromic stimulation experiments which entails stimulation of proximal neurons as well.

Given our conditions that include the use of mature animals for slice experiments and a requirement for long-lasting 
recordings that are typically associated with intracellular dialysis, we could not couple biocytin fillings with post hoc immunocytochemistry (e.g. Kawaguchi and Kubota 1996; Kawaguchi and Kondo 2002) for determining the neurochemistry of LTS interneurons. A more fruitful approach proved to be using PV- and SOM- cre mice crossed with td-tomato lines to obtain targeted recordings from PV and SOM interneurons: This allowed us to make inferences about the neurochemistry of the interneuron involved in feedback inhibition. PV interneurons in the LA with one exception (1/9) did not possess feedback interneuron properties. On the other hand, SOM+interneurons which exhibited electrophysiological similarities with LTS interneurons all exhibited spiking activity in response to BL stimulation suggesting that they receive local inputs from LA principal neurons. One pitfall in our interpretation concerns the lack of spikes in LTS interneurons during paired recordings as these neurons were recorded around a membrane potential of $-70 \mathrm{mV}$ for consistency purposes. In this respect, experiments utilizing voltage sensitive dyes or calcium sensors are required for a more definitive conclusion. Nonetheless, it is important to re-iterate that LTS interneurons constituted the major cell type responsive to the excitation of principal neurons during paired recordings. These findings are in line with studies from cortex where LTS neurons constitute the SOM+ Martinotti cells serving vital functions in feedback inhibition (Kawaguchi and Kubota 1996; Goldberg et al. 2004; Wang et al. 2004; Ma et al. 2006). The absence of such responses in PV interneurons rules out the possibility of a potential contamination from the stimulation of extra-amygdalar inputs. Furthermore, SOM+interneurons resembling LTS interneurons were reciprocally connected to principal neurons at a very high rate and the uIPSPs they evoked had slow rise times, consistent with the notion that these feedback interneurons are dendrite targeting interneurons (Muller et al. 2007; Fino and Yuste 2011). Last but not the least, the feedback LTS interneurons observed in our recordings from both wild type and SOM-tdtomato crosses received facilitating synapses from principal neurons, a recurring motif in other cortical regions where SOM+ Martinotti cells serve as feedback interneurons (Thomson and Dechars 1997; Markram et al. 1998; Wang et al. 2004).

A number of anatomical studies have found that $\mathrm{PV}+$ interneurons receive inputs from local BLA principal neurons in different species while receiving minimal extra-amygdalar input (Smith et al. 2000; Woodruff and Sah 2007; Unal et al. 2014; Spampanato et al. 2016) while no direct data exists for SOM+ interneurons. These anatomical studies receive support by Woodruff and Sah (2007) who observed around $27 \%$ connectivity in the principal cell to $\mathrm{PV}+$ interneuron direction in the BLA while we observed 1 out of $9 \mathrm{PV}+$ interneurons responsive to the activation of local LA circuitry as a 
result of BL stimulation. This suggests a lower portion of $\mathrm{PV}+$ interneurons $(11 \%)$ serve as feedback interneurons in the LA. The discrepancy with our study could be accounted by a number of factors either in isolation or interacting with each other. First of all, we do not have direct information about the neurochemistry of LTS feedback cells we recorded in wild type mice. A proportion of these cells could well be PV+. A second interesting possibility pertains to subtle differences of the network architecture of LA and other BLA nuclei: we have exclusively recorded in LA neurons while the entire BLA was covered by Woodruff and Sah (2007). Our observation of $\mathrm{PV}$ immunoreactivity in SOM-tomato cells in the BL but not in the LA potentially signifies the different developmental history of PV and SOM interneurons in these structures. A third possibility pertains to the age of animals as Woodruff and Sah (2007) used younger (PD 16-25) mice while we used PD 30-60 mice.

Another factor pertaining to species differences could underline the discrepancy of the current results with anatomical studies (Smith et al. 2000; Unal et al. 2014). These studies point out to the notion that $\mathrm{PV}+$ cells might not be contributing as much to feedforward inhibition while studies done in rats have shown that fast-spiking interneurons, which are typically PV+ in the BLA as well, constitute a responsive population to extra-amygdalar stimulation (Szinyei et al. 2000; Sosulina et al. 2010; Spampanato et al. 2011). It is important to note that, our experiments do not negate the possibility of SOM+interneurons exerting feedforward inhibition (Unal et al. 2014) and other functions (McDonald et al. 2012; Capogna 2014) as is the case in the neocortex (Beierlein et al. 2003; Cruikshank et al. 2010).

The current study adds to the repertoire of potential functions, such as disinhibition (Wolff et al. 2014) and long-range projections (McDonald et al. 2012), for SOM+interneurons. Feedback inhibition is a circuit mechanism thought to be critical for the formation of specific engrams within the amygdala (Kim et al. 2013; 2016). Furthermore, the dendritic location of feedback interneuron inputs on principal neurons elsewhere endows these neurons with the capability of regulating excitatory inputs to the level of single dendritic branches and even spines (Golding et al. 2002; Kampa et al. 2006; Humeau and Luthi 2007; Lovett-Barron et al. 2012; Bar Ilan et al. 2013; Kim et al. 2013, 2016; Cichon and Gan 2015) determining the exact locus of synaptic plasticity and formation of specific memories (Cichon and Gan 2015. In essence, the abovementioned mechanisms might be instrumental in parsing the LA into distinct sensory/functional compartments as is the case in other cortical regions (e.g. Fino and Yuste 2011; Adesnik et al. 2012; Zhang et al. 2014).
This study concludes that LTS interneurons which are predominantly $\mathrm{SOM}+$ perform feedback inhibition functions in the LA. Further optogenetic studies are required to obtain data with higher throughput in the LA and other BLA nuclei along with functional studies testing their implications.

Acknowledgements Open Access funding provided by Projekt DEAL. This work was supported by the Max Planck Florida Institute.

\section{Compliance with ethical standards}

Conflict of interest The authors do not have any conflict of interest.

Ethical statement This study was performed in strict accordance with the recommendations in the Guide for the Care and Use of Laboratory Animals of the National Institutes of Health, and all animals were handled according to protocols approved by the Institutional Animal Care and Use Committee of the Max Planck Florida Institute for Neuroscience.

Open Access This article is licensed under a Creative Commons Attribution 4.0 International License, which permits use, sharing, adaptation, distribution and reproduction in any medium or format, as long as you give appropriate credit to the original author(s) and the source, provide a link to the Creative Commons licence, and indicate if changes were made. The images or other third party material in this article are included in the article's Creative Commons licence, unless indicated otherwise in a credit line to the material. If material is not included in the article's Creative Commons licence and your intended use is not permitted by statutory regulation or exceeds the permitted use, you will need to obtain permission directly from the copyright holder. To view a copy of this licence, visit http://creativecommons.org/licenses/by/4.0/.

\section{References}

Adesnik H, Bruns W, Taniguchi H, Huang ZJ, Scanziani M (2012) A neural circuit for spatial summation in visual cortex. Nature 490(7419):226

Bar-Ilan L, Gidon A, Segev I (2013) The role of dendritic inhibition in shaping the plasticity of excitatory synapses. Front Neural Circ 6:118

Beierlein M, Gibson JR, Connors BW (2003) Two dynamically distinct inhibitory networks in layer 4 of the neocortex. J Neurophysiol 90(5):2987-3000

Bienvenu TC, Busti D, Magill PJ, Ferraguti F, Capogna M (2012) Cell-type-specific recruitment of amygdala interneurons to hippocampal theta rhythm and noxious stimuli in vivo. Neuron 74(6):1059-1074

Butler RK, Oliver EM, Fadel JR, Wilson MA (2018) Hemispheric differences in the number of parvalbumin-positive neurons in subdivisions of the rat basolateral amygdala complex. Brain Res 1678:214-219

Capogna M (2014) GABAergic cell type diversity in the basolateral amygdala. Curr Opin Neurobiol 26:110-116

Cauli B, Porter JT, Tsuzuki K, Lambolez B, Rossier J, Quenet B, Audinat E (2000) Classification of fusiform neocortical interneurons based on unsupervised clustering. Proc Natl Acad Sci 97(11):6144-6149

Cichon J, Gan WB (2015) Branch-specific dendritic Ca 2+ spikes cause persistent synaptic plasticity. Nature 520(7546):180 
Cruikshank SJ, Urabe H, Nurmikko AV, Connors BW (2010) Pathway-specific feedforward circuits between thalamus and neocortex revealed by selective optical stimulation of axons. Neuron 65(2):230-245

Faber ESL, Callister RJ, Sah P (2001) Morphological and electrophysiological properties of principal neurons in the rat lateral amygdala in vitro. J Neurophysiol 85(2):714-723

Fee C, Banasr M, Sibille E (2017) Somatostatin-positive gammaaminobutyric acid interneuron deficits in depression: cortical microcircuit and therapeutic perspectives. Biological psychiatry, 82(8):549-559

Fino E, Yuste R (2011) Dense inhibitory connectivity in neocortex. Neuron 69(6): 1188-1203

Forloni G, Hohmann C, Coyle JT (1990) Developmental expression of somatostatin in mouse brain. I Immunocytochemical studies. Develop Brain Res 53(1):6-25

Gaudreau H, Pare D (1996a) Projection neurons of the lateral amygdaloid nucleus are virtually silent throughout the sleep-waking cycle. J Neurophysiol 75(3):1301-1305

Gibson JR, Beierlein M, Connors BW (1999) Two networks of electrically coupled inhibitory neurons in neocortex. Nature 402(6757):75

Goldberg JH, Lacefield CO, Yuste R (2004) Global dendritic calcium spikes in mouse layer 5 low threshold spiking interneurones: implications for control of pyramidal cell bursting. J Physiol 558(2):465-478

Golding NL, Staff NP, Spruston N (2002) Dendritic spikes as a mechanism for cooperative long-term potentiation. Nature 418(6895):326

Hu H, Cavendish JZ, Agmon A (2013) Not all that glitters is gold: offtarget recombination in the somatostatin-IRES-Cre mouse line labels a subset of fast-spiking interneurons. Front Neural Circ 7:195

Humeau Y, Lüthi A (2007) Dendritic calcium spikes induce bi-directional synaptic plasticity in the lateral amygdala. Neuropharmacology 52(1):234-243

Kampa BM, Letzkus JJ, Stuart GJ (2006) Requirement of dendritic calcium spikes for induction of spike-timing-dependent synaptic plasticity. J Physiol 574(1):283-290

Kawaguchi Y, Kondo S (2002) Parvalbumin, somatostatin and cholecystokinin as chemical markers for specific GABAergic interneuron types in the rat frontal cortex. J Neurocytol 31(3-5):277-287

Kawaguchi Y, Kubota Y (1996) Physiological and morphological identification of somatostatin-or vasoactive intestinal polypeptidecontaining cells among GABAergic cell subtypes in rat frontal cortex. J Neurosci 16(8):2701-2715

Kim D, Pare D, Nair SS (2013) Assignment of model amygdala neurons to the fear memory trace depends on competitive synaptic interactions. J Neurosci 33(36):14354-14358

Kim D, Samarth P, Feng F, Pare D, Nair SS (2016) Synaptic competition in the lateral amygdala and the stimulus specificity of conditioned fear: a biophysical modeling study. Brain Struct Funct 221(4):2163-2182

Krabbe S, Gründemann J, Lüthi A (2018) Amygdala inhibitory circuits regulate associative fear conditioning. Biol Psychiatry 83(10):800-809

LeDoux JE (2000) Emotion circuits in the brain. Annu Rev Neurosci 23(1):155-184

Letzkus JJ, Wolff SB, Lüthi A (2015) Disinhibition, a circuit mechanism for associative learning and memory. Neuron 88(2):264-276

Lovett-Barron M, Turi GF, Kaifosh P, Lee PH, Bolze F, Sun XH, Losonczy A et al (2012) Regulation of neuronal input transformations by tunable dendritic inhibition. Nature Neurosci 15(3):423

Lucas EK, Jegarl AM, Morishita H, Clem RL (2016) Multimodal and site-specific plasticity of amygdala parvalbumin interneurons after fear learning. Neuron 91(3):629-643
Ma Y, Hu H, Berrebi AS, Mathers PH, Agmon A (2006) Distinct subtypes of somatostatin-containing neocortical interneurons revealed in transgenic mice. J Neurosci 26(19):5069-5082

Markram H, Wang Y, Tsodyks M (1998) Differential signaling via the same axon of neocortical pyramidal neurons. Proc Natl Acad Sci 95(9):5323-5328

Marowsky A, Rudolph U, Fritschy JM, Arand M (2012) Tonic inhibition in principal cells of the amygdala: a central role for $\alpha 3$ subunit-containing GABAA receptors. J Neurosci 32(25):8611-8619

McDonald AJ (1992) Projection neurons of the basolateral amygdala: a correlative Golgi and retrograde tract tracing study. Brain Res Bull 28(2):179-185

McDonald AJ (1998) Cortical pathways to the mammalian amygdala. Progress Neurobiol 55(3):257-332

McDonald AJ, Augustine JR (1993) Localization of GABA-like immunoreactivity in the monkey amygdala. Neuroscience 52(2):281-294

McDonald AJ, Augustine JR (2019) Nonpyramidal neurons in the primate basolateral amygdala: a Golgi study in the baboon (Papio cynocephalus) and long-tailed macaque (Macaca fascicularis). J Comparat Neurol 528:772-786

McDonald AJ, Betette RL (2001) Parvalbumin-containing neurons in the rat basolateral amygdala: morphology and co-localization of Calbindin-D28k. Neuroscience 102(2):413-425

McDonald AJ, Mascagni F (2001) Colocalization of calcium-binding proteins and GABA in neurons of the rat basolateral amygdala. Neuroscience 105(3):681-693

McDonald AJ, Mascagni F (2002) Immunohistochemical characterization of somatostatin containing interneurons in the rat basolateral amygdala. Brain research 943(2):237-244

McDonald AJ, Mascagni F, Zaric V (2012) Subpopulations of somatostatin-immunoreactive non-pyramidal neurons in the amygdala and adjacent external capsule project to the basal forebrain: evidence for the existence of GABAergic projection neurons in the cortical nuclei and basolateral nuclear complex. Front Neural Circ 6:46

Muller JF, Mascagni F, McDonald AJ (2007) Postsynaptic targets of somatostatin-containing interneurons in the rat basolateral amygdala. J Comparat Neurol 500(3):513-529

Papadopoulos GC, Cavanagh ME, Antonopoulos J, Michaloudi H, Parnavelas JG (1993) Postnatal development of somatostatincontaining neurons in the visual cortex of normal and darkreared rats. Exp Brain Res 92(3):473-478

Pape HC, Pare D (2010) Plastic synaptic networks of the amygdala for the acquisition, expression, and extinction of conditioned fear. Physiol Rev 90(2):419-463

Paré D, Smith Y (1993) Distribution of GABA immunoreactivity in the amygdaloid complex of the cat. Neuroscience 57(4):1061-1076

Popescu AT, Paré D (2010) Synaptic interactions underlying synchronized inhibition in the basal amygdala: evidence for existence of two types of projection cells. J Neurophysiol 105(2):687-696

Rainnie DG, Mania I, Mascagni F, McDonald AJ (2006) Physiological and morphological characterization of parvalbumin-containing interneurons of the rat basolateral amygdala. J Comparat Neurol 498(1):142-161

Samson RD, Dumont ÉC, Paré D (2003) Feedback inhibition defines transverse processing modules in the lateral amygdala. J Neurosci 23(5):1966-1973

Smith Y, Paré JF, Paré D (2000) Differential innervation of parvalbumin-immunoreactive interneurons of the basolateral amygdaloid complex by cortical and intrinsic inputs. J Comparat Neurol 416(4):496-508 
Sosulina L, Graebenitz S, Pape HC (2010) GABAergic interneurons in the mouse lateral amygdala: a classification study. J Neurophysiol 104(2):617-626

Spampanato J, Polepalli J, Sah P (2011) Interneurons in the basolateral amygdala. Neuropharmacology 60(5):765-773

Spampanato J, Sullivan RK, Perumal MB, Sah P (2016) Development and physiology of GABAergic feedback excitation in parvalbumin expressing interneurons of the mouse basolateral amygdala. Physiol Rep 4:1

Swanson LW, Petrovich GD (1998) What is the amygdala? Trends Neurosci 21(8):323-331

Szinyei C, Heinbockel T, Montagne J, Pape HC (2000) Putative cortical and thalamic inputs elicit convergent excitation in a population of GABAergic interneurons of the lateral amygdala. J Neurosci 20(23):8909-8915

Thomson AM, Deuchars J (1997) Synaptic interactions in neocortical local circuits: dual intracellular recordings in vitro. Cerebral Cortex (N Y: 1991) 7(6):510-522

Truitt WA, Johnson PL, Dietrich AD, Fitz SD, Shekhar A (2009) Anxiety-like behavior is modulated by a discrete subpopulation of interneurons in the basolateral amygdala. Neuroscience 160(2):284-294

Unal G, Paré JF, Smith Y, Paré D (2014) Cortical inputs innervate calbindin-immunoreactive interneurons of the rat basolateral amygdaloid complex. J Comp Neurol 522(8):1915-1928. https:// doi.org/10.1002/cne.23511

Urban-Ciecko J, Barth AL (2016) Somatostatin-expressing neurons in cortical networks. Nature Rev Neurosci 17(7):401
Vereczki VK, Veres JM, Müller K, Nagy GA, Rácz B, Barsy B, Hájos N (2016) Synaptic organization of perisomatic GABAergic inputs onto the principal cells of the mouse basolateral amygdala. Front Neuroanatomy 10:20

Wang Y, Toledo-Rodriguez M, Gupta A, Wu C, Silberberg G, Luo J, Markram H (2004) Anatomical, physiological and molecular properties of Martinotti cells in the somatosensory cortex of the juvenile rat. J Physiol 561(1):65-90

Washburn MS, Moises HC (1992) Electrophysiological and morphological properties of rat basolateral amygdaloid neurons in vitro. J Neurosci 12(10):4066-4079

Wolff SB, Gründemann J, Tovote P, Krabbe S, Jacobson GA, Müller C, Lüthi A et al (2014) Amygdala interneuron subtypes control fear learning through disinhibition. Nature 509(7501):453

Woodruff AR, Sah P (2007) Networks of parvalbumin-positive interneurons in the basolateral amygdala. J Neurosci 27(3):553-563

Yavorska I, Wehr M (2016) Somatostatin-expressing inhibitory interneurons in cortical circuits. Front Neural Circ 10:76

Zhang S, Xu M, Kamigaki T, Do JPH, Chang WC, Jenvay S, Dan Y et al (2014) Long-range and local circuits for top-down modulation of visual cortex processing. Science 345(6197):660-665

Publisher's Note Springer Nature remains neutral with regard to jurisdictional claims in published maps and institutional affiliations. 